\title{
Dynamic Advertising under Vertical Product Differentiation*
}

\author{
Luca Colombo ${ }^{\dagger}$ - Luca Lambertini ${ }^{\ddagger}$
}

14 December, 2002

\begin{abstract}
We investigate a dynamic advertising model where product quality is endogenous. In the differential game between single-product firms, there exists a parameter range where the low-quality firm uses a more efficient advertising technology and earns higher profits than the rival. Moreover, we show that equilibrium qualities are the same under duopoly, multiproduct monopoly and social planning, the only distortion being concerned with output levels.

Keywords: advertising, product quality, differential games, optimal control.

JEL classification: L12, L13, M37, C73.
\end{abstract}

${ }^{*}$ We thank Gustav Feichtinger for an insightful discussion that inspired this paper, Roberto Cellini and the seminar audience in Bologna for useful comments. The usual disclaimer applies.

${ }^{\dagger}$ Research Fellow, Department of Economics, University of Bologna, Strada Maggiore 45, 40125 Bologna, Italy; fax +39-51-6402664; e-mail: colombo@spbo.unibo.it.

${ }^{\ddagger}$ Professor of Economics, Department of Economics, University of Bologna, Strada Maggiore 45, 40125 Bologna, Italy; fax +39-51-6402664; e-mail: lamberti@spbo.unibo.it. 


\section{Introduction}

There exists a wide literature dealing with dynamic advertising either in monopoly or in oligopoly models (see, e.g., Jørgensen, 1982; Erickson, 1991; Feichtinger et al., 1994; Dockner et al., ch. 11). To the best of our knowledge, the endogenous interplay between advertising and product quality has not received a large amount of attention so far. The few existing contributions in this field usually consider the optimal control of advertising efforts and product quality in relation with building up the stock of goodwill, under incomplete consumer information. ${ }^{1}$ One interesting exception dealing with a full information model of dynamic advertising and product quality is in Ouardighi and Pasin (2002), extending the well known Lanchester model to account for the interplay between market shares and quality. ${ }^{2}$ Others have investigated, adopting either static or dynamic approaches, the strategic use of product qualities as firms' instruments to build up market shares (Moorthy, 1988; Motta, 1993; Dutta et al., 1995).

We study persuasive advertising in a dynamic market where fully informed consumers may choose between two goods characterised by different quality levels which are endogenously determined. We consider (i) the differential game between two single-product firms; (ii) the optimal control problem faced by a monopolist supplying both varieties so as to maximise profits, and (iii) the optimal control problem solved by a benevolent planner supplying both varieties so as to maximise social welfare.

Our main results can be summarised as follows. In the duopoly game, there exist parameter ranges wherein the low-quality firm earns higher profits than the high-quality firm, due to the fact that the number of consumers choosing the lowquality good is considerably larger than the number of those who buy the high-quality good. This is in contrast with the acquired wisdom coming from the existing static games describing quality competition in oligopoly (Gabszewicz and Thisse, 1979, 1980; Shaked and Sutton, 1982, 1983; Lehmann-Grube, 1997, inter alia), where the possible interaction between quality and advertising is completely disregarded and the equilibrium market share of the high-quality good is always larger than the lowquality good's. While in the static literature on this issue it is always true that supplying a superior quality enables the firm (i) to extract a higher profit margin from each unit and (ii) to obtain a larger market share than rivals', here we show that being able to attract the richest set of costumers is insufficient to ensure higher profits because inferior qualities may serve larger market shares. This appears to be realistic, e.g. if one considers that selling a luxury motorbike like Ducati 999 may well entail a larger unit price-cost margin than selling a Honda Hornet, but the number

\footnotetext{
${ }^{1}$ See Schmalensee (1978), Kotowitz and Mathewson (1979), Conrad (1985), Feichtinger et al. (1994, and the references therein).

${ }^{2}$ For the formulation of the Lanchester model, see Dockner et al. (2000, ch. 11), Case (1979), Sorger (1989) and Erickson (1991).
} 
of customers that can afford a Hornet is surely much larger than the number of those who may afford a Ducati 999.

Moreover, in our model the quality levels turn out to be independent of the market regime, i.e., they are the same at the duopoly, monopoly, and planning equilibria. Again, this finds no correspondence in the static literature on vertical product differentiation, where it usually emerges that the degree of vertical differentiation at the duopoly equilibrium is larger than the monopoly optimum and the social optimum, due to the incentive for firms to increase product differentiation so as to soften price competition (Shaked and Sutton, 1982; Champsaur and Rochet, 1989). In addition to this, our result also tells that there is no quality distortion at the monopoly equilibrium, as compared to the first best. While in static models we observe the incentive for the monopolist to under- or oversupply product quality in order to induce selfdiscrimination across consumers (Spence, 1975; Mussa and Rosen, 1978; Champsaur and Rochet, 1989), here we only observe a downward distortion in output levels due to monopoly power.

The remainder of the paper is structured as follows. The basic setup is laid out in section 2 . The duopoly setting is investigated in section 3, while section 4 contains the analysis of the monopoly and first best equilibria. Concluding remarks are in section 5 .

\section{The Model}

Traditionally, models dealing with vertically differentiated duopoly postulate the existence of a consumers' gross surplus function which is assumed to be non-separable in its arguments. Instead, we assume that all the arguments of the utility function are additively separable. Consumers are indexed by a marginal willingness to pay $\theta \in\left[0, \theta_{1}\right]$ with density equal to one, so that the total mass of consumers is $\theta_{1}$. The distribution of consumers and the support are invariant w.r.t. time. The market exists for $t \in[0, \infty)$, with time being considered as continuous. At each $t$, the market is supplied by two single-product firms offering goods of quality $q_{i}(t)$ with $i=\{H, L\}$, $q_{H}(t) \geq q_{L}(t) \geq 0$, which are perfectly observable, together with the price vector, by consumers before purchase. Production entails an instantaneous variable cost of quality improvement, which is assumed to be convex in the current quality level:

$$
C_{i}(t)=c_{i} x_{i}(t)\left[q_{i}(t)\right]^{2}
$$

with constant parameters $0<c_{H}<c_{L}$ indicating that the high quality firm is more efficient in the production of quality.

From the consumption of the high quality good, a consumer of type $\theta$ draws the following net surplus:

$$
U_{H}=\theta+q_{H}(t)-p_{H}(t)
$$


where $p_{H}(t)$ is the market price of variety $H$ at time $t$. Similarly, from the consumption of the low quality good, a consumer indexed by $\theta$ draws the following net surplus:

$$
U_{L}=s \theta+q_{L}(t)-p_{L}(t)
$$

where $p_{L}(t)$ is the market price of variety $L$ at time $t$, and $s \in(0,1)$ is a positive and time-invariant parameter capturing the idea that gross satisfaction of such consumer from buying the low quality is lower. If a consumer does not buy either variety, the resulting utility is nil. In order to obtain the expressions of market demands, we compute the threshold of $\theta$ which characterizes the consumer who is indifferent between buying from the high quality firm and buying from the low quality firm:

$$
\widehat{\theta}(t)=\frac{p_{H}(t)-p_{L}(t)-q_{H}(t)+q_{L}(t)}{1-s}
$$

and the analogous threshold of $\theta$ characterizing the consumer who is indifferent between buying from the low quality firm and not buying at all:

$$
\tilde{\theta}(t)=\frac{p_{L}(t)-q_{L}(t)}{s}
$$

The direct demand system follows:

$$
\begin{gathered}
x_{H}(t)=\theta_{1}-\widehat{\theta}(t) \\
x_{L}(t)=\widehat{\theta}(t)-\widetilde{\theta}(t)
\end{gathered}
$$

provided that $\tilde{\theta}(t)>0$, ensuring that we are in the non-degenerate case where partial market coverage prevails. If so, we can write the inverse demand system:

$$
\begin{aligned}
& p_{H}(t)=\theta_{1}+q_{H}(t)-x_{H}(t)-s x_{L}(t) \\
& p_{L}(t)=q_{L}(t)+s\left[\theta_{1}-x_{H}(t)-x_{L}(t)\right]
\end{aligned}
$$

Observe that expressions (4-9) correctly define the demand system if and only if $\widehat{\theta}(t) \geq \widetilde{\theta}(t)$, which amounts to requiring that $s\left[p_{H}(t)-q_{H}(t)\right] \geq\left[p_{L}(t)-q_{L}(t)\right]$. In the remainder of the paper, we will check that this condition indeed holds at the duopoly steady state equilibrium. The economic interpretation of this requirement is that one should exclude the case of quality leapfrogging in either direction, i.e., any situation such that the firm endowed with a technological efficiency parameter $c_{H}$ (respectively, $c_{L}$ ) decides to produce a lower (resp., higher) quality than the firm using the less (resp., more) efficient technology. 
Instantaneous profits write as follows:

$$
\pi_{i}(t)=\left[p_{i}(t)-c_{i}\left[q_{i}(t)\right]^{2}\right] x_{i}(t)-b_{i}\left[a_{i}(t)\right]^{2}
$$

where $b_{i}\left[a_{i}(t)\right]^{2}$ is the instantaneous cost of investing in advertising, $a_{i}(t)$ being the advertising effort of firm $i$ at time $t$. We assume that sales evolve over time in response to advertising investments, according to the following kinematic equation (as in Vidale and Wolfe, 1957):

$$
\frac{\partial x_{i}(t)}{\partial t} \equiv \dot{x}_{i}=a_{i}(t)-\delta x_{i}(t) \quad i=H, L
$$

where $\delta$ denotes the depreciation (disaffection) rate, constant over time and common to both firms. It is worth stressing that this advertising technology has asymmetric effects on the market demand of the two firms. The reason is that the low-quality firm may increase demand by attracting consumers in the lower part of the preference spectrum (i.e., pushing $\widetilde{\theta}(t)$ down) as well as in the intermediate range of preferences (i.e., pushing $\widehat{\theta}(t)$ up), while the high-quality firm can only fight against the rival for $\widehat{\theta}(t)$, since the upper bound of the willingness to pay, $\theta_{1}$, is given. This preludes to the possibility for the low-quality firm to perform better than the high-quality firm in equilibrium.

Firm $i$ aims at maximising the discounted profit flow:

$$
\Pi_{i}(t)=\int_{0}^{\infty} \pi_{i}(t) e^{-\rho t}
$$

w.r.t. controls $q_{i}(t)$ and $a_{i}(t)$, under the constraint given by the state dynamics (11).

For future reference, we also define consumer surplus:

$$
C S(t)=C S_{L}(t)+C S_{H}(t)=\int_{\tilde{\theta}(t)}^{\widehat{\theta}(t)}\left(s z+q_{L}(t)-p_{L}(t)\right) d z+\int_{\widehat{\theta}(t)}^{\theta_{1}}\left(z+q_{H}(t)-p_{H}(t)\right) d z
$$

where

$$
C S_{L}(t)=\frac{s\left[x_{L}(t)\right]^{2}}{2} ; C S_{H}(t)=\frac{x_{H}(t)}{2}\left[x_{H}(t)+2 s x_{L}(t)\right]
$$

If we disregard the issue of surplus distribution between consumers and producers, and confine our attention to Pareto efficiency, we can define welfare as follows:

$$
W(t)=C S_{H}(t)+C S_{L}(t)+\pi_{H}(t)+\pi_{L}(t) .
$$




\section{Duopoly Equilibrium}

Firm $i^{\prime}$ s current value Hamiltonian function is:

$$
\mathcal{H}_{i}(t)=e^{-\rho t}\left[\pi_{i}(t)+\lambda_{i i}(t) \dot{x}_{i}+\lambda_{i j}(t) \dot{x}_{j}\right]
$$

First order conditions (FOCs) on controls are (henceforth, we omit the indication of time for brevity): ${ }^{3}$

$$
\begin{gathered}
\frac{\partial \mathcal{H}_{i}}{\partial q_{i}}=0 \Rightarrow x_{i}\left(1-2 c_{i} q_{i}\right)=0 \Rightarrow q_{i}=\frac{1}{2 c_{i}} \\
\frac{\partial \mathcal{H}_{i}}{\partial a_{i}}=0 \Rightarrow-2 a_{i} b_{i}+\lambda_{i i}=0 \Rightarrow \lambda_{i i}=2 a_{i} b_{i} \Rightarrow \dot{\lambda}_{i i}=2 \dot{a}_{i} b_{i}
\end{gathered}
$$

The above FOCs entail that the present game is a 'linear state game', producing subgame perfect (or Markov perfect) open-loop Nash equilibria. ${ }^{4}$ Notice also that condition (17-18) do not contain $\lambda_{i j}$ because the present game features separated dynamics. ${ }^{5}$ Therefore, the problem admits the solution $\lambda_{i j}=0$ for all $t \in[0, \infty)$ and $j \neq i$. Accordingly we specify only one co-state equation per firm:

$$
\begin{gathered}
\frac{\partial \mathcal{H}_{H}}{\partial x_{H}}=-\delta \lambda_{H H}+\theta_{1}+q_{H}-c_{H} q_{H}^{2}-2 x_{H}-s x_{L}=\rho \lambda_{H H}-\dot{\lambda}_{H H} \\
\frac{\partial \mathcal{H}_{L}}{\partial x_{L}}=-\delta \lambda_{L L}+q_{L}-c_{L} q_{L}^{2}+s\left(\theta_{1}-x_{H}-2 x_{L}\right)=\rho \lambda_{L L}-\dot{\lambda}_{L L}
\end{gathered}
$$

along with the transversality condition:

$$
\lim _{t \rightarrow \infty} \mu_{i}(t) x_{i}(t)=0
$$

and the initial conditions $x_{i}(0)>0$.

Now, using (18) and the co-state equations, we write:

$$
\dot{a}_{H}=\frac{\dot{\lambda}_{H H}}{2 b_{H}}=-\frac{1}{4 c_{H}}-\theta_{1}+2 a_{H} b_{H}(\delta+\rho)+2 x_{H}+s x_{L}
$$

\footnotetext{
${ }^{3}$ Second order conditions are always met throughout the paper. They are omitted for brevity.

${ }^{4}$ See, e.g., Leitmann and Schmitendorf (1978) and Feichtinger (1983). For an exhaustive exposition of linear state games, see Dockner et al. (2000, chapter 7).

${ }^{5}$ This also entails that, rewriting the model over a finite time horizon with an appropriate scrap value, this problem could also be solved with the alternative coordinate transformation methos of Dockner and Leitmann (2001).
} 


$$
\dot{a}_{L}=\frac{\dot{\lambda}_{L L}}{2 b_{L}}=-\frac{1}{4 c_{L}}+2 a_{L} b_{L}(\delta+\rho)+s\left(-\theta_{1}+x_{H}+2 x_{L}\right)
$$

The steady state equilibrium requires $\dot{a}_{H}=0, \dot{a}_{L}=0$, yielding:

$$
\begin{aligned}
& a_{H}=\frac{1+4 c_{H}\left(\theta_{1}-2 x_{H}-s x_{L}\right)}{8 b_{H} c_{H}(\delta+\rho)} \\
& a_{L}=\frac{1+4 c_{L} s\left(\theta_{1}-x_{H}-2 x_{L}\right)}{8 b_{L} c_{L}(\delta+\rho)}
\end{aligned}
$$

which can be plugged into the system of state equations (11), simplifying as follows:

$$
\begin{gathered}
\dot{x}_{H}=\frac{1+4 c_{H}\left(\theta_{1}-2 x_{H}-s x_{L}\right)}{8 b_{H} c_{H}(\delta+\rho)}-\delta x_{H} \\
\dot{x}_{L}=\frac{1+4 c_{L} s\left(\theta_{1}-x_{H}-2 x_{L}\right)}{8 b_{L} c_{L}(\delta+\rho)}-\delta x_{L}
\end{gathered}
$$

We are interested in investigating the dynamics of the system in the positive quadrant of the space $\left\{x_{i}, a_{i}\right\}$, which is described in figure 1 . The system $\left\{\dot{x}_{i}=0, \dot{a}_{i}=0\right\}$ yields the following steady state sales:

$$
\begin{aligned}
x_{H}^{S S} & =\frac{2 b_{L} c_{L} \delta\left(1+4 c_{H} \theta_{1}\right)(\delta+\rho)-s\left[-2 c_{L}+c_{H}\left(1+4 c_{L} \theta_{1}(s-2)\right)\right]}{4 c_{H} c_{L}\left(4 b_{L} \delta(\delta+\rho)\left(1+b_{H} \delta(\delta+\rho)+\left(4+4 b_{H} \delta(\delta+\rho)-s\right) s\right)\right.} \\
x_{L}^{S S} & =\frac{-c_{L} s+2 c_{H}\left(1+2 c_{L} \theta_{1} s+b_{H} \delta(\delta+\rho)\left(1+4 c_{L} \theta_{1} s\right)\right)}{4 c_{H} c_{L}\left(4 b_{L} \delta(\delta+\rho)\left(1+b_{H} \delta(\delta+\rho)+\left(4+4 b_{H} \delta(\delta+\rho)-s\right) s\right)\right.}
\end{aligned}
$$

These expressions can be plugged into (24-25) to yield the optimal investments in advertising, $a_{i}^{S S}$. The following holds:

Proposition 1 The steady state defined by $\left\{a_{i}^{S S}, x_{i}^{S S}\right\}$ is a saddle point.

Proof. See the Appendix.

The above output levels are acceptable if and only if they are non-negative. This of course depends upon the relative size of demand and cost parameters, $\left\{s, \theta_{1}, b_{i}, c_{i}\right\}$. First note that the denominator of $x_{i}^{S S}$ is positive for all admissible values of parameters. Since we are treating the issue of enlarging market shares through advertising, it is interesting to examine the non-negativity of outputs in terms of parameters $b_{i}$ and their interaction with production cost parameters $c_{i}$. We can prove: 
Lemma 2 The equilibrium output of the high-quality firm, $x_{H}^{S S}$, is always positive.

Proof. Examine the numerator of (28). The first term

$$
2 b_{L} c_{L} \delta\left(1+4 c_{H} \theta_{1}\right)(\delta+\rho)
$$

is clearly positive. Then, it can be quickly established that:

$$
-s\left[-2 c_{L}+c_{H}\left(1+4 c_{L} \theta_{1}(s-2)\right)\right]>0
$$

as well. To see this, rewrite it as:

$$
s\left[\left(2 c_{L}-c_{H}\right)+4 c_{H} c_{L} \theta_{1}(2-s)\right]
$$

which is positive for all $s \in(0,1)$ and $c_{H}<c_{L}$.

Now examine the output of the low-quality firm:

Lemma 3 If

$$
c_{H} \in\left(\frac{c_{L} s}{2\left(1+2 c_{L} \theta_{1} s\right)}, c_{L}\right),
$$

then $x_{L}^{S S}>0$ for all $b_{H}>0$. If instead

$$
c_{H} \in\left(0, \frac{c_{L} s}{2\left(1+2 c_{L} \theta_{1} s\right)}\right],
$$

then $x_{L}^{S S}>0$ for all

$$
b_{H}>\bar{b}_{H} \equiv \frac{s c_{L}-2 c_{H}\left(1+2 c_{L} \theta_{1} s\right)}{2 \delta c_{H}(\rho+\delta)\left(1+4 \theta_{1} c_{L} s\right)} \geq 0 .
$$

Otherwise, $x_{L}^{S S}=0$ for all $b_{H} \in\left[0, \bar{b}_{H}\right]$.

Proof. In general, $x_{L}^{S S}>0$ for all

$$
b_{H}>\bar{b}_{H} \equiv \frac{s c_{L}-2 c_{H}\left(1+2 c_{L} \theta_{1} s\right)}{2 \delta c_{H}(\rho+\delta)\left(1+4 \theta_{1} c_{L} s\right)} .
$$

This is surely true for all admissible $b_{H}$ if

$$
c_{H} \in\left(\frac{c_{L} s}{2\left(1+2 c_{L} \theta_{1} s\right)}, c_{L}\right),
$$

since this implies $\bar{b}_{H}<0$. In the case where

$$
c_{H} \in\left(0, \frac{c_{L} s}{2\left(1+2 c_{L} \theta_{1} s\right)}\right],
$$

then $\bar{b}_{H} \geq 0$. Therefore, in this range of $c_{H}$, we have that $x_{L}^{S S} \geq 0$ iff $b_{H} \geq \bar{b}_{H}$. This concludes the proof.

Now, on the basis of Lemma 3, one can also check that, in equilibrium, the non negativity of $x_{L}^{S S} \equiv \widehat{\theta}(t)-\widetilde{\theta}(t)$ also ensures that $\widehat{\theta}(t) \geq \widetilde{\theta}(t)$. Lemmata 2-3 directly imply a relevant Corollary: 


\section{Corollary 4 If}

$$
c_{H} \in\left(0, \frac{c_{L} s}{2\left(1+2 c_{L} \theta_{1} s\right)}\right]
$$

then the high-quality firm is a monopolist for all $b_{H} \in\left[0, \bar{b}_{H}\right]$.

When the efficiency of the advertising technology of firm $H$ is very high (or, conversely, the cost of advertising is sufficiently low), there is no room in the market for the inferior variety.

For (28-29) to be acceptable, it must also be that $\theta_{1}>x_{H}^{S S}+x_{L}^{S S}$, i.e., firms indeed cover the market only partially:

Lemma $5 \theta_{1}>x_{H}^{S S}+x_{L}^{S S}$ for all $b_{H}>\max \left\{\underline{b}_{H}, 0\right\}$, where:

$$
\underline{b}_{H} \equiv \frac{c_{L}\left[s+2 b_{L} \delta(\delta+\rho)\right]+c_{H}\left[2-8 b_{L} c_{L} \delta \theta_{1}(\delta+\rho)-s\left(1+4 c_{L} \theta_{1}\right)\right]}{2 c_{H} \delta(\delta+\rho)\left[4 c_{L} \theta_{1}\left(2 b_{L} \delta(\delta+\rho)+s\right)-1\right]}
$$

with $\partial \underline{b}_{H} / \partial b_{L}<0$ always.

Proof. The derivation of $\underline{b}_{H}$ is straigthforward. Then observe that:

$$
\frac{\partial \underline{b}_{H}}{\partial b_{L}}=-\frac{c_{L}\left[1+4 c_{H} \theta_{1}(1-s)\right]}{c_{H}\left[4 c_{L} \theta_{1}\left(2 b_{L} \delta(\delta+\rho)+s\right)-1\right]^{2}}<0 .
$$

The above result entails that, intuitively, partial market coverage is easier to obtain in equilibrium, the higher the level of both advertising cost parameters. Now define as

$$
\widehat{b}_{H} \equiv \frac{2 b_{L} c_{L} \delta(\delta+\rho)+3 c_{L} s-c_{H}\left(2+s+4 c_{L} \theta_{1}\left(-2 b_{L} \delta(\delta+\rho)+(-1+s) s\right)\right)}{2 c_{H} \delta(\delta+\rho)\left(1+4 c_{L} \theta_{1} s\right)}
$$

the level of $b_{H}$ such that $x_{H}^{S S}=x_{L}^{S S}$. Assessing $x_{H}^{S S}-x_{L}^{S S}$ one can easily prove: ${ }^{6}$

Lemma 6 If $\widehat{b}_{H}>0$, then $x_{H}^{S S}>x_{L}^{S S}$ for all $b_{H} \in\left(0, \widehat{b}_{H}\right) ; x_{H}^{S S}<x_{L}^{S S}$ for all $b_{H}>\widehat{b}_{H}$. If instead $\widehat{b}_{H} \leq 0$, then $x_{L}^{S S}>x_{H}^{S S}$.

The foregoing discussion (in particular, Corollary 4 and Lemma 6) opens the possibility that there exist admissible parameter constellations such that both firms enjoy positive market shares and profits in equilibrium, with the low-quality firm serving more consumers and earning higher profits than the high-quality firm.

\footnotetext{
${ }^{6}$ As we already know, for all $s \in(0,1)$, the denominator of $x_{i}^{S S}$ is always positive. Therefore, the value of $b_{H}$ such that $x_{H}^{S S}-x_{L}^{S S}=0$ is unique and it is given by $\widehat{b}_{H}$.
} 
We now turn to the comparative statics on the steady state level of sales w.r.t. all parameters. First, the property $\partial x_{i}^{S S} / \partial c_{j}>0, i \neq j$, can be easily interpreted. All else equal, as firm $j$ becomes less efficient in supplying quality (i.e., $q_{j}$ decreases as $c_{j}$ increases), the product of firm $j$ becomes less appealing and some customers switch to firm $i$. With regard to an increase in the own cost of quality improvement, we have the expected property $\partial x_{i}^{S S} / \partial c_{i}<0$. Not surprisingly, $\partial x_{i}^{S S} / \partial \theta_{1}>0$. That is, as the market becomes more affluent, demand for all varieties increases.

Now consider the effects of a change in the efficiency of the advertising investments on market shares. First of all, notice that

$$
\frac{\partial x_{H}^{S S}}{\partial b_{H}}<0 ; \frac{\partial x_{L}^{S S}}{\partial b_{H}}>0
$$

in the whole admissible parameter range. This is what one would expect from the outset: as the advertising campaign of firm $H$ becomes less expensive (or more effective), the demand for the high-quality good is enhanced while that for the inferior variety shrinks.

The effect of a change in $b_{L}$ on $x_{i}^{S S}$ is more involved:

$$
\begin{aligned}
\frac{\partial x_{H}^{S S}}{\partial b_{L}} & >0 \text { and } \frac{\partial x_{L}^{S S}}{\partial b_{L}}<0 \text { for all } c_{H}>\widetilde{c}_{H} \\
\widetilde{c}_{H} & =\frac{s c_{L}}{2\left[1+2 c_{L} \theta_{1} s+b_{H} \delta\left(1+4 c_{L} \theta_{1} s\right)(\delta+\rho)\right]}
\end{aligned}
$$

and conversely for all $c_{H} \in\left(0, \widetilde{c}_{H}\right]$. This means that, when vertical product differentiation is relatively low (which holds when $c_{H}>\widetilde{c}_{H}$ ), any increase in $b_{L}$ drive some consumers to switch from the inferior variety to the high-quality good. This does not happen if the degree of differentiation is large enough.

We are now in a position to assess firms' relative performance in terms of steady state profits:

$\pi_{H}^{S S}=\frac{\left(1+b_{H} \delta(\delta+2 \rho)\right)\left[-2 b_{L} c_{L} \delta\left(1+4 c_{H} \theta_{1}\right)(\delta+\rho)-2 c_{L}+c_{H}\left(1+4 c_{L} \theta_{1}(-2+s)\right) s\right]^{2}}{16 c_{H}^{2} c_{L}^{2}\left[4 b_{L} \delta(\delta+\rho)\left(1+b_{H} \delta(\delta+\rho)\right)+\left(4+4 b_{H} \delta(\delta+\rho)-s\right) s\right]^{2}}$

$$
\pi_{L}^{S S}=\frac{\left(b_{L} \delta(\delta+2 \rho)+s\right)\left[c_{L} s-2 c_{H}\left(1+2 c_{L} \theta_{1} s+b_{H} \delta(\delta+\rho)\left(1+4 c_{L} \theta_{1} s\right)\right)\right]^{2}}{16 c_{H}^{2} c_{L}^{2}\left[4 b_{L} \delta(\delta+\rho)\left(1+b_{H} \delta(\delta+\rho)\right)+\left(4+4 b_{H} \delta(\delta+\rho)-s\right) s\right]^{2}}
$$

By evaluating the sign of the difference between $\pi_{H}^{S S}$ and $\pi_{L}^{S S}$, we obtain a critical value $\widetilde{b}_{H}$ at which $\pi_{H}^{S S}=\pi_{L}^{S S}$ entailing the following: ${ }^{7}$

\footnotetext{
${ }^{7}$ The equation $\pi_{H}^{S S}=\pi_{L}^{S S}$ yields two roots. While the smaller one is always negative, the larger may or may not be positive. The expressions are omitted for brevity.
} 
Lemma 7 If $\widetilde{b}_{H}>0$, then $\pi_{H}^{S S}>\pi_{L}^{S S}$ for all $b_{H} \in\left(0, \widetilde{b}_{H}\right) ; \pi_{H}^{S S}<\pi_{L}^{S S}$ for all $b_{H}>\widetilde{b}_{H}$. If instead $\widetilde{b}_{H} \leq 0$, then $\pi_{L}^{S S}>\pi_{H}^{S S}$.

Moreover, comparing $\widehat{b}_{H}$ against $\bar{b}_{H}$, we obtain:

Lemma $8 \widehat{b}_{H}>\bar{b}_{H}$ always.

Proof. It suffices to observe that

$$
\widehat{b}_{H}-\bar{b}_{H} \propto 2 b_{L} c_{L} \delta\left(1+4 c_{H} \theta_{1}\right)(\delta+\rho)-s\left[-2 c_{L}+c_{H}\left(1+4 c_{L} \theta_{1}(s-2)\right)\right]
$$

with the expression of the r.h.s. coinciding with the numerator of (28), that, as we already know from Lemma 2 , is always positive.

Lemmata 6-8 yield a partition of the parameter space into three regions, according to the value assumed by the advertising efficiency parameter of high quality firm, $b_{H}$. This partition is illustrated in figure 1, assuming the parameter set is such that $\widetilde{b}_{H}>\widehat{b}_{H}>0>\max \left\{\underline{b}_{H}, \bar{b}_{H}\right\} .8$ This means that we exclude the trivial case where the market is a monopoly for firm $H$.

$$
\begin{array}{rll}
a_{H}^{S S}>a_{L}^{S S} & a_{H}^{S S}<a_{L}^{S S} & a_{H}^{S S}<a_{L}^{S S} \\
x_{H}^{S S}>x_{L}^{S S} & x_{H}^{S S}<x_{L}^{S S} & x_{H}^{S S}<x_{L}^{S S} \\
\pi_{H}^{S S}>\pi_{L}^{S S} & \pi_{H}^{S S}>\pi_{L}^{S S} & \pi_{H}^{S S}<\pi_{L}^{S S} \\
\widehat{\widehat{b}}_{H} & \widetilde{b}_{H} & b_{H}
\end{array}
$$

Figure 1 : Parameter Space

Proposition 9 Take the values of $\left\{s, \theta_{1}, c_{i}\right\}$ such that $\widetilde{b}_{H}>\widehat{b}_{H}>0$. If $b_{H} \in\left(0, \widehat{b}_{H}\right)$, then $x_{H}^{S S}>x_{L}^{S S}, a_{H}^{S S}>a_{L}^{S S}$ and $\pi_{H}^{S S}>\pi_{L}^{S S}$. If $b_{H} \in\left(\widehat{b}_{H}, \widetilde{b}_{H}\right)$, then $x_{H}^{S S}<x_{L}^{S S}, a_{H}^{S S}$ $<a_{L}^{S S}$ and $\pi_{H}^{S S}>\pi_{L}^{S S}$. If $b_{H}>\widetilde{b}_{H}$, then $x_{H}^{S S}<x_{L}^{S S}, a_{H}^{S S}<a_{L}^{S S}$ and $\pi_{H}^{S S}<\pi_{L}^{S S}$.

The above Proposition illustrates a situation where parameter values are such that there exists an admissible ranges wherein both firms are active and earn positive profits in steady state. Moreover, in contrast with the conclusions commonly drawn from the static approach to vertical differentiation in oligopoly, in one subset of parameters the low-quality firm performs better than the high-quality firm in terms of both market share and equilibrium profits. This fact can be interpreted as follows.

\footnotetext{
${ }^{8}$ One such numerical example is in the Appendix.
} 
First of all, consider that the critical levels $\widetilde{b}_{H}$ and $\widehat{b}_{H}$ are a function of $b_{L}$. Then, examine the case $b_{H} \in\left(0, \widehat{b}_{H}\right)$. Here, the advertising technology of firm $H$ is sufficiently efficient, as compared to that of firm $L$, to imply that firm $H$ acquires a dominant position in the market. If instead $b_{H} \in\left(\widehat{b}_{H}, \widetilde{b}_{H}\right)$, the relative decrease in the efficiency of her advertising campaign induces firm $H$ to invest less in advertising, which, in turn, entails that her equilibrium demand becomes lower than firm $L$ 's. This, however, is not yet sufficient to reverse the inequality on profits because the reduction in the market share is still more than offset by the quality differential. In the third range, where $b_{H}>\widetilde{b}_{H}$, the distribution of consumers between firms induces a reversal of the profit ranking. All this amounts to saying that having a larger market share in equilibrium is not sufficient to earn higher profits than the rival.

Observe that, in line of principle, $\widehat{b}_{H}>\widetilde{b}_{H}$ is admissible. However, this case would not allow for a sensible interpretation in terms of the underlying economics. In particular, if that were indeed the case, one would observe a non-monotone behaviour of the relative profits of firms. In particular, as the advertising technology of firm $H$ becomes less efficient, we would obtain that initially $\pi_{H}^{S S}>\pi_{L}^{S S}$, then $\pi_{H}^{S S}<\pi_{L}^{S S}$ for $b_{H} \in\left(\widetilde{b}_{H}, \widehat{b}_{H}\right)$, then again $\pi_{H}^{S S}>\pi_{L}^{S S}$ for $b_{H}>\widehat{b}_{H}$. For this reason, we can dismiss such a case.

\section{Monopoly and Social Planning}

Now we examine the regimes where, respectively, both plants are controlled by (i) a profit-seeking monopolist, and (ii) a benevolent planner maximising social welfare. In the monopoly regime $(M)$, the current value Hamiltonian function turns out to be:

$$
\mathcal{H}^{M}=e^{-\rho t}\left(\pi_{H}+\pi_{L}+\lambda_{H} \dot{x}_{H}+\lambda_{L} \dot{x}_{L}\right)
$$

while under social planning $(S P)$, it writes:

$$
\mathcal{H}^{S P}=e^{-\rho t}\left(W+\lambda_{H} \dot{x}_{H}+\lambda_{L} \dot{x}_{L}\right)
$$

where the instantaneous social welfare function $W$ is defined as in (15).

The first relevant result is stated in the following:

Proposition 10 Under both monopoly and social planning, optimal qualities are the same as under the profit-seeking duopoly $(D)$ :

$$
q_{i}^{k}=\frac{1}{2 c_{i}}, i=H, L, k=D, M, S P .
$$

Proof. To show this, it suffices to observe that, irrespective of the property structure of the two plants (i.e., of the regime $k$ ), the first order condition on the Hamiltonian 
w.r.t. quality $q_{i}$ is:

$$
\frac{\partial \mathcal{H}^{k}}{\partial q_{i}}=\frac{\partial \mathcal{H}_{i}^{D}}{\partial q_{i}}=\frac{\partial \pi_{i}}{\partial q_{i}}=0
$$

since consumer surplus (14) is independent of quality levels.

Another obvious result needs no proof. This regards the profit performance of the industry in the three regimes at stake. Clearly, the highest attainable profits in equilibrium accrue to the monopolist selling both varieties, that, by definition, must perform better than the sum of two independent single-good duopolists. Then, it is also straightforward that the profit-seeking duopolists earn strictly larger profits than a social planner taking care of consumer surplus into his objective function.

Concerning the performance of the monopolist selling both varieties, we can prove:

Proposition 11 Under monopoly, the optimal advertising efforts for generic output levels are:

$$
a_{H}^{M}=\frac{1+4 c_{H}\left(\theta_{1}-2 x_{H}-2 s x_{L}\right)}{8 b_{H} c_{H}(\delta+\rho)} ; a_{L}^{M}=\frac{1+4 c_{L} s\left(\theta_{1}-2 x_{H}-2 x_{L}\right)}{8 b_{L} c_{L}(\delta+\rho)}
$$

while steady state output levels are:

$$
\begin{aligned}
x_{H}^{M} & =\frac{b_{L} c_{L} \delta\left(1+4 c_{H} \theta_{1}\right)(\delta+\rho)+s\left(c_{L}+c_{H}\left(-1+4 c_{L} \theta_{1}-4 c_{L} s \theta_{1}\right)\right)}{8 c_{H} c_{L}\left(b_{L} \delta(\delta+\rho)\left(1+b_{H} \delta(\delta+\rho)+\left(1+b_{H} \delta(\delta+\rho)-s\right) s\right)\right.} \\
x_{L}^{M} & =\frac{-c_{L} s+c_{H}\left(1+b_{H} \delta(\delta+\rho)\left(1+4 c_{L} \theta_{1} s\right)\right)}{8 c_{H} c_{L}\left(b_{L} \delta(\delta+\rho)\left(1+b_{H} \delta(\delta+\rho)+\left(1+b_{H} \delta(\delta+\rho)-s\right) s\right)\right.} .
\end{aligned}
$$

The monopoly equilibrium is a saddle point.

Proof. See the Appendix.

If a planner controls both firms, we obtain:

Proposition 12 Under social planning, the optimal advertising efforts for generic output levels are:

$$
a_{H}^{S P}=\frac{1+4 c_{H}\left(\theta_{1}-x_{H}-s x_{L}\right)}{8 b_{H} c_{H}(\delta+\rho)} ; a_{L}^{S P}=\frac{1+4 c_{L} s\left(\theta_{1}-x_{H}-x_{L}\right)}{8 b_{L} c_{L}(\delta+\rho)}
$$

while steady state output levels are:

$$
\begin{aligned}
x_{H}^{S P} & =\frac{2 b_{L} c_{L} \delta\left(1+4 c_{H} \theta_{1}\right)(\delta+\rho)+s\left(c_{L}+c_{H}\left(-1+4 c_{L} \theta_{1}-4 c_{L} s \theta_{1}\right)\right)}{4 c_{H} c_{L}\left(2 b_{L} \delta(\delta+\rho)\left(1+2 b_{H} \delta(\delta+\rho)+\left(1+2 b_{H} \delta(\delta+\rho)-s\right) s\right)\right.} \\
x_{L}^{S P} & =\frac{-c_{L} s+c_{H}\left(1+2 b_{H} \delta(\delta+\rho)\left(1+4 c_{L} \theta_{1} s\right)\right)}{4 c_{H} c_{L}\left(2 b_{L} \delta(\delta+\rho)\left(1+2 b_{H} \delta(\delta+\rho)+\left(1+2 b_{H} \delta(\delta+\rho)-s\right) s\right)\right.} .
\end{aligned}
$$

The social optimum is a saddle point. 
Proof. See the Appendix.

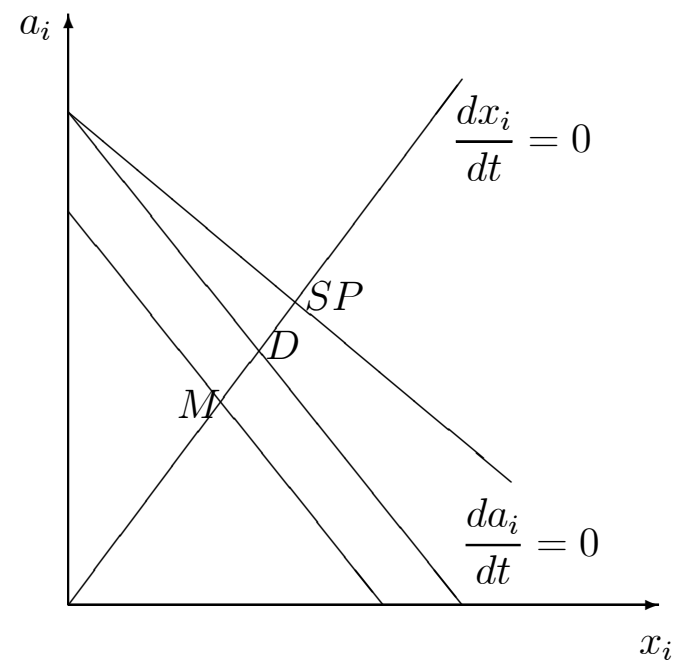

Figure 2 : Comparisons

One last remark is in order, concerning the different incentives to invest in advertising in the three regimes. The state dynamics (11) (the same across regimes) and the dynamics of advertising efforts $a_{i}$ (changing across regimes) are drawn in figure 2. Without further proof, the properties of this graph, together with the appropriate expressions of optimal advertising efforts in the three regimes, produce the following Proposition.

Proposition 13 For a given $x_{j}$ it follows that $a_{i}^{S P}>a_{i}^{D}>a_{i}^{M}$ implying that $x_{i}^{S P}>$ $x_{i}^{D}>x_{i}^{M}$.

As the planner aims at maximising the sum of profits and consumer surplus, and qualities are the same across regimes, then clearly the social incentive to invest in advertising is driven by the fact that increasing the extent of market coverage amounts to increasing welfare. This also explains that, in both profit-seeking regimes, which by definition do not take into account consumer surplus, the advertising expenditure must be lower than the socially desirable investment. Moreover, if each variety is supplied by an independent firm, she surely invests more to advertise her product than a monopolist would do for the same variety. The reason is that a single-product duopolist wants to steal costumers from the demand basin of the rival, which of course cannot be the case for a multiproduct monopolist. 


\section{Concluding Remarks}

We have proposed a differential duopoly game where each firm may invest both in product quality and advertising campaigns. We have assumed that sales evolve over time in response of advertising investments, while product quality improvements do not require any capital accumulation to take place. Contrary to the results we are familiar with from static analyses, we have shown that there exists a range of parameters in which the low-quality firm gains a higher market share as well as higher profits than the rival. The reason behind this result lies in the relative efficiency of the advertising technologies used by the two firms.

Moreover, we have shown that the level of product quality provided by a duopoly corresponds to the one which is socially desirable. This is true also under a monopolistic regime. The unique distortions which arise at the privately optimal equilibria are on the output side. These downward distortions due to market power are obviously induced by pricing above marginal cost and are therefore qualitatively the same as in the static literature. 


\section{Appendix}

Proof of Proposition 1. We consider first the system composed by (11) in combination with the appropriate kinematics of the control variable $a_{i}$, that is, (22) or (23), alternatively. In the two cases, that can be treated in isolation because of the separated dynamics assumed in the model, the system can be written in matrix form as follows:

$$
\begin{aligned}
& {\left[\begin{array}{c}
\dot{x}_{H} \\
\dot{a}_{H}
\end{array}\right]=\left[\begin{array}{cc}
-\delta & 1 \\
2 & 2 b_{H}(\delta+\rho)
\end{array}\right]\left[\begin{array}{l}
x_{H} \\
a_{H}
\end{array}\right]+\left[\begin{array}{c}
0 \\
s x_{L}-\frac{1}{4 c_{H}}-\theta_{1}
\end{array}\right]} \\
& {\left[\begin{array}{c}
\dot{x}_{L} \\
\dot{a}_{L}
\end{array}\right]=\left[\begin{array}{cc}
-\delta & 1 \\
2 s & 2 b_{L}(\delta+\rho)
\end{array}\right]\left[\begin{array}{c}
x_{L} \\
a_{L}
\end{array}\right]+\left[\begin{array}{c}
0 \\
s x_{H}-\frac{1}{4 c_{L}}-s \theta_{1}
\end{array}\right]}
\end{aligned}
$$

Since the determinants of the above $2 \times 2$ matrices are both negative, the equilibria we have obtained are two saddles. From the phase diagram, it is clear that these can be approached only along the north-west arm of the saddle path.

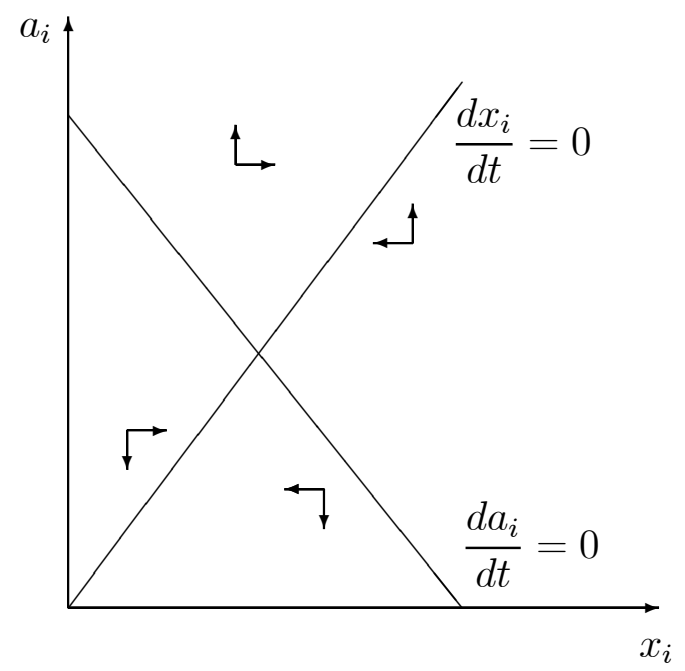

Figure 3 : Phase Diagram

Numerical duopoly example. Take the following numerical values of parameters:

$$
\theta_{1}=1 ; b_{L}=\frac{1}{10} ; c_{H}=\frac{7}{10} ; c_{L}=\frac{3}{4} ; s=\frac{9}{10} ; \delta=\rho=\frac{1}{20} \text {. }
$$

If so, then $\pi_{L}^{S S}>\pi_{H}^{S S}$ for all $b_{H}>\widetilde{b}_{H} \simeq 22.105$. Setting $b_{H}=22.3$, the numerical values of equilibrium variables are:

$$
\pi_{H}^{S S} \simeq 0.2037 ; \pi_{L}^{S S} \simeq 0.2041 ; x_{H}^{S S} \simeq 0.4178 ; x_{L}^{S S}=0.4760 .
$$


Proof of Proposition 11. First order conditions relative to the Hamiltonian (35) are:

$$
\begin{gathered}
\frac{\partial \mathcal{H}^{M}}{\partial q_{i}}=0 \Rightarrow x_{i}-2 c_{i} q_{i} x_{i}=0 \Rightarrow q_{i}=\frac{1}{2 c_{i}} \\
\frac{\partial \mathcal{H}^{M}}{\partial a_{i}}=0 \Rightarrow-2 a_{i} b_{i}+\lambda_{i}=0 \Rightarrow \lambda_{i}=2 a_{i} b_{i} \Rightarrow \dot{\lambda}_{i}=2 \dot{a}_{i} b_{i} \\
\frac{\partial \mathcal{H}^{M}}{\partial x_{H}}=-\delta \lambda_{H}+\theta_{1}+q_{H}-c_{H} q_{H}^{2}-2 x_{H}-2 s x_{L}=\rho \lambda_{H}-\dot{\lambda}_{H} \\
\frac{\partial \mathcal{H}^{M}}{\partial x_{L}}=-\delta \lambda_{L}+q_{L}-c_{L} q_{L}^{2}+s\left(\theta_{1}-2 x_{H}-2 x_{L}\right)=\rho \lambda_{L}-\dot{\lambda}_{L}
\end{gathered}
$$

along with the same initial and transversality conditions as in duopoly.

From (a5) and (a6):

$$
\begin{aligned}
& \dot{\lambda}_{H}=(\delta+\rho) \lambda_{H}-\theta_{1}-q_{H}+c_{H} q_{H}^{2}+2 x_{H}+2 s x_{L} \\
& \dot{\lambda}_{L}=(\delta+\rho) \lambda_{L}-q_{L}+c_{L} q_{L}^{2}-s\left(\theta_{1}-2 x_{H}-2 x_{L}\right)
\end{aligned}
$$

while from (a4):

$$
\begin{gathered}
\dot{a}_{H}=\frac{\dot{\lambda}_{H}}{2 b_{H}}=-\frac{1}{4 c_{H}}-\theta_{1}+2 a_{H} b_{H}(\delta+\rho)+2 x_{H}+2 s x_{L} \\
\dot{a}_{L}=\frac{\dot{\lambda}_{L}}{2 b_{L}}=-\frac{1}{4 c_{L}}+2 a_{L} b_{L}(\delta+\rho)+s\left(-\theta_{1}+2 x_{H}+2 x_{L}\right) .
\end{gathered}
$$

The steady state equilibrium requires $\left\{\dot{a}_{H}=0, \dot{a}_{L}=0\right\}$ :

$$
a_{H}^{M}=\frac{1+4 c_{H}\left(\theta_{1}-2 x_{H}-2 s x_{L}\right)}{8 b_{H} c_{H}(\delta+\rho)} ; a_{L}^{M}=\frac{1+4 c_{L} s\left(\theta_{1}-2 x_{H}-2 x_{L}\right)}{8 b_{L} c_{L}(\delta+\rho)}
$$

Then, simplifying the kinematics of states yields:

$$
\dot{x}_{H}=\frac{1+4 c_{H}\left(\theta_{1}-2 x_{H}-2 s x_{L}\right)}{8 b_{H} c_{H}(\delta+\rho)}-\delta x_{H}
$$




$$
\dot{x}_{L}=\frac{1+4 c_{L} s\left(\theta_{1}-2 x_{H}-2 x_{L}\right)}{8 b_{L} c_{L}(\delta+\rho)}-\delta x_{L}
$$

At the resulting unique steady state, sales are:

$$
\begin{gathered}
x_{H}^{S S}=\frac{b_{L} c_{L} \delta\left(1+4 c_{H} \theta_{1}\right)(\delta+\rho)+s\left(c_{L}+c_{H}\left(-1+4 c_{L} \theta_{1}-4 c_{L} s \theta_{1}\right)\right)}{8 c_{H} c_{L}\left(b_{L} \delta(\delta+\rho)\left(1+b_{H} \delta(\delta+\rho)+\left(1+b_{H} \delta(\delta+\rho)-s\right) s\right)\right.} \\
x_{L}^{S S}=\frac{-c_{L} s+c_{H}\left(1+b_{H} \delta(\delta+\rho)\left(1+4 c_{L} \theta_{1} s\right)\right)}{8 c_{H} c_{L}\left(b_{L} \delta(\delta+\rho)\left(1+b_{H} \delta(\delta+\rho)+\left(1+b_{H} \delta(\delta+\rho)-s\right) s\right)\right.}
\end{gathered}
$$

The dynamic system can be written in matrix form:

$$
\left[\begin{array}{l}
\dot{x}_{H} \\
\dot{a}_{H} \\
\dot{x}_{L} \\
\dot{a}_{L}
\end{array}\right]=\left[\begin{array}{llll}
-\delta & 1 & 0 & 0 \\
2 & 2 b_{H}(\delta+\rho) & 2 s & 0 \\
0 & 0 & -\delta & 1 \\
2 s & 0 & 2 s & 2 b_{L}(\delta+\rho)
\end{array}\right]\left[\begin{array}{c}
x_{H} \\
a_{H} \\
x_{L} \\
a_{L}
\end{array}\right]+\left[\begin{array}{c}
0 \\
-\frac{1}{4 c_{H}}-\theta_{1} \\
0 \\
1 \\
-\frac{1}{4 c_{L}}-s \theta_{1}
\end{array}\right]
$$

By computing the four eigenvalues, it is easy to assess that: $\lambda_{1}=\lambda_{3}>0$ and $\lambda_{2}=\lambda_{4}<0$. Hence the equilibrium is a saddle point.

Proof of Proposition 12. First order conditions relative to the social planner's Hamiltonian (36) are:

$$
\begin{gathered}
\frac{\partial \mathcal{H}^{S P}}{\partial q_{i}}=0 \Rightarrow x_{i}-2 c_{i} q_{i} x_{i}=0 \Rightarrow q_{i}=\frac{1}{2 c_{i}} \\
\frac{\partial \mathcal{H}^{S P}}{\partial a_{i}}=0 \Rightarrow-2 a_{i} b_{i}+\lambda_{i i}=0 \Rightarrow \lambda_{i i}=2 a_{i} b_{i} \Rightarrow \dot{\lambda}_{i i}=2 \dot{a}_{i} b_{i} \\
\frac{\partial \mathcal{H}^{S P}}{\partial x_{H}}=-\delta \lambda_{H}+\theta_{1}+q_{H}-c_{H} q_{H}^{2}-x_{H}-s x_{L}=\rho \lambda_{H H}-\dot{\lambda}_{H H} \\
\frac{\partial \mathcal{H}^{S P}}{\partial x_{L}}=-\delta \lambda_{L L}+q_{L}-c_{L} q_{L}^{2}+s\left(\theta_{1}-x_{H}-x_{L}\right)=\rho \lambda_{L L}-\dot{\lambda}_{L L}
\end{gathered}
$$

with the same initial and transversality conditions as in the previous cases.

From (a18) and (a19):

$$
\dot{\lambda}_{H}=(\delta+\rho) \lambda_{H}-\theta_{1}-q_{H}+c_{H} q_{H}^{2}+x_{H}+s x_{L}
$$




$$
\dot{\lambda}_{L}=(\delta+\rho) \lambda_{L}-q_{L}+c_{L} q_{L}^{2}-s\left(\theta_{1}-x_{H}-x_{L}\right)
$$

while from (a17):

$$
\begin{gathered}
\dot{a}_{H}=\frac{\dot{\lambda}_{H}}{2 b_{H}}=-\frac{1}{4 c_{H}}-\theta_{1}+2 a_{H} b_{H}(\delta+\rho)+x_{H}+s x_{L} \\
\dot{a}_{L}=\frac{\dot{\lambda}_{L}}{2 b_{L}}=-\frac{1}{4 c_{L}}+2 a_{L} b_{L}(\delta+\rho)+s\left(-\theta_{1}+x_{H}+x_{L}\right)
\end{gathered}
$$

The steady state equilibrium requirement $\left\{\dot{a}_{H}=0, \dot{a}_{L}=0\right\}$ yields:

$$
a_{H}^{S P}=\frac{1+4 c_{H}\left(\theta_{1}-x_{H}-s x_{L}\right)}{8 b_{H} c_{H}(\delta+\rho)} ; a_{L}^{S P}=\frac{1+4 c_{L} s\left(\theta_{1}-x_{H}-x_{L}\right)}{8 b_{L} c_{L}(\delta+\rho)}
$$

that can be used to rewrite the state equations:

$$
\begin{gathered}
\dot{x}_{H}=\frac{1+4 c_{H}\left(\theta_{1}-x_{H}-s x_{L}\right)}{8 b_{H} c_{H}(\delta+\rho)}-\delta x_{H} \\
\dot{x}_{L}=\frac{1+4 c_{L} s\left(\theta_{1}-x_{H}-x_{L}\right)}{8 b_{L} c_{L}(\delta+\rho)}-\delta x_{L}
\end{gathered}
$$

Solving the system $\left\{\dot{x}_{H}=0, \dot{x}_{L}=0\right\}$, one obtains the steady state sales:

$$
\begin{aligned}
x_{H}^{S S} & =\frac{2 b_{L} c_{L} \delta\left(1+4 c_{H} \theta_{1}\right)(\delta+\rho)+s\left(c_{L}+c_{H}\left(-1+4 c_{L} \theta_{1}-4 c_{L} s \theta_{1}\right)\right)}{4 c_{H} c_{L}\left(2 b_{L} \delta(\delta+\rho)\left(1+2 b_{H} \delta(\delta+\rho)+\left(1+2 b_{H} \delta(\delta+\rho)-s\right) s\right)\right.} \\
x_{L}^{S S} & =\frac{-c_{L} s+c_{H}\left(1+2 b_{H} \delta(\delta+\rho)\left(1+4 c_{L} \theta_{1} s\right)\right)}{4 c_{H} c_{L}\left(2 b_{L} \delta(\delta+\rho)\left(1+2 b_{H} \delta(\delta+\rho)+\left(1+2 b_{H} \delta(\delta+\rho)-s\right) s\right)\right.}
\end{aligned}
$$

Finally, the dynamic system can be written in matrix form, as follows:

$$
\left[\begin{array}{l}
\dot{x}_{H} \\
\dot{a}_{H} \\
\dot{x}_{L} \\
\dot{a}_{L}
\end{array}\right]=\left[\begin{array}{llll}
-\delta & 1 & 0 & 0 \\
1 & 2 b_{H}(\delta+\rho) & s & 0 \\
0 & 0 & -\delta & 1 \\
s & 0 & s & 2 b_{L}(\delta+\rho)
\end{array}\right]\left[\begin{array}{c}
x_{H} \\
a_{H} \\
x_{L} \\
a_{L}
\end{array}\right]+\left[\begin{array}{c}
0 \\
-\frac{1}{4 c_{H}}-\theta_{1} \\
0 \\
-\frac{1}{4 c_{L}}-s \theta_{1}
\end{array}\right]
$$

Again, computing the four eigenvalues one can check that: $\lambda_{1}=\lambda_{3}>0$ and $\lambda_{2}=\lambda_{4}<0$. Therefore, the equilibrium is a saddle point. 


\section{References}

[1] CASE, J., Economics and the Competitive Process, New York University Press, New York, 1979.

[2] CHAMPSAUR, P., and ROCHET, J.-C., Multiproduct Duopolists, Econometrica, Vol. 57, pp. 533-557, 1989.

[3] CONRAD, K., Quality, Advertising and the Formation of Goodwill under Dynamic Conditions, Optimal Control Theory and Economic Analysis, edited by G. Feichtinger, North-Holland, Amsterdam, Vol. 2, pp. 215-234, 1985.

[4] DOCKNER, E.J. and LEITMANN, G., Coordinate Transformations and Derivation of Open-Loop Nash Equilibria, Journal of Optimization Theory and Applications, Vol. 110, pp. 1-15, 2001.

[5] DOCKNER, E.J, JØRGENSEN, S., LONG, N.V. and SORGER, G., Differential Games in Economics and Management Science, Cambridge University Press, Cambridge, 2000.

[6] DUTTA, P.K., LACH, S. and RUSTICHINI, A., Better Late than Early: Vertical Differentiation in the Adoption of a New Technology, Journal of Economics and Management Strategy, Vol. 4, pp. 563-589, 1995.

[7] ERICKSON, G.M., Dynamic Models of Advertising Competition, Kluwer, Dordrecht, 1991.

[8] FEICHTINGER, G., The Nash Solution of an Advertising Differential Game: Generalization of a Model by Leitmann and Schmitendorf, IEEE Transactions on Automatic Control, Vol. 28, pp. 1044-1048, 1983.

[9] FEICHTINGER, G., HARTL, R.F. and SETHI, P.S., Dynamic Optimal Control Models in Advertising: Recent Developments, Management Science, Vol. 40, 195226, 1994.

[10] GABSZEWICZ, J.J. and THISSE J.-F, Price Competition, Quality and Income Disparities, Journal of Economic Theory, Vol. 20, pp. 340-59, 1979.

[11] GABSZEWICZ, J.J. and THISSE J.-F, Entry (and Exit) in a Differentiated Industry, Journal of Economic Theory, Vol. 22, pp. 327-338, 1980.

[12] JØRGENSEN, S., A Survey of Some Differential Games in Advertising, Journal of Economics Dynamics and Control, Vol. 4, pp. 341-369, 1982.

[13] KOTOWITZ, Y. and MATHEWSON F., Advertising, Consumer Information, and Product Quality, Bell Journal of Economics, Vol. 10, pp. 566-588, 1979. 
[14] LEHMANN-GRUBE, U., Strategic Choice of Quality when Quality is Costly: The Persistence of the High-Quality Advantage, RAND Journal of Economics, Vol. 28, pp. 372-384, 1997.

[15] LEITMANN, G. and SCHMITENDORF W.E., Profit Maximization through Advertising: A Nonzero Sum Differential Game Approach, IEEE Transactions on Automatic Control, Vol. 23, pp. 646-650, 1978.

[16] MOORTHY, K.S., Product and Price Competition in a Duopoly Model, Marketing Science, Vol. 7, pp. 141-168, 1988

[17] MOTTA, M., Endogenous Quality Choice: Price vs Quantity Competition, Journal of Industrial Economics, Vol. 41, pp. 113-32, 1993.

[18] MUSSA, M., and ROSEN S., Monopoly and Product Quality, Journal of Economic Theory, Vol. 18, pp. 301-317, 1978.

[19] OUARDIGHI, F.E., and PASIN, F., Advertising and Quality Decisions under Dynamic Conditions, X International Symposium on Dynamic Games and Applications, State University of St. Petersburg, July 8-11, 2002.

[20] SCHMALENSEE, R., A Model of Advertising and Product Quality. Journal of Political Economy, Vol. 86, pp. 1213-1225, 1978.

[21] SHAKED, A. and SUTTON J., Relaxing Price Competition through Product Differentiation, Review of Economic Studies, Vol. 69, pp. 3-13, 1982.

[22] SHAKED, A. and SUTTON J., Natural Oligopolies, Econometrica, Vol. 51, pp. 1469-83, 1983.

[23] SORGER, G., Competitive Dynamic Advertising: A Modification of the Case Game, Journal of Economic Dynamics and Control, Vol. 13, pp. 55-80, 1989.

[24] SPENCE, A.M., Monopoly, Quality and Regulation, Bell Journal of Economics, Vol. 6, pp. 417-429, 1975.

[25] VIDALE, M.L. and WOLFE, H.B., An Operations Research Study of Sales Response to Advertising, Operations Research, Vol. 5, pp. 370-381, 1957. 